\title{
SCATTERED COMPACTIFICATIONS AND THE ORDERABILITY OF SCATTERED SPACES. II
}

\author{
S. PURISCH
}

\begin{abstract}
A space is suborderable if it is embeddable in a (totally) orderable space. It is shown that a suborderable scattered space is orderable and admits an orderable scattered compactification.
\end{abstract}

1. Introduction. A space is suborderable (also called generalized orderable) if it is embeddable in a (totally) orderable space, and it is scattered if each of its nonempty subsets has an isolated point. The length of a scattered space $X$ is the least ordinal $\alpha$ such that the $\alpha$ th derived set $X^{(\alpha)}$ of $X$ is empty.

In 1976 it was announced $\left[\mathbf{P}_{\mathbf{1}}\right]$ that every suborderable scattered space of countable length is orderable and admits an orderable scattered compactification, and it was conjectured that the countable length condition could be eliminated. The proof $\left[\mathbf{P}_{3}\right]$ involved a simple induction. To extend the proof to a space of uncountable length a transfinite induction was necessary, and the major stumbling block was to insure that the proof did not break down at limit stages. After talking, in 1980, with R. Telgarsky it became apparent that paracompactness was the key to pass through limit stages using the type of reduction principles found in [T]. But many suborderable scattered spaces are not paracompact. It was discovered, however, that at limit stages, such a space can be partitioned into open sets, each of which satisfies a paracompactness-like property away from an end gap, which makes these sets manageable. This is the central idea in the following proof, and because of it the proof here is actually simpler at limit stages than that of the special case in $\left[\mathbf{P}_{\mathbf{3}}\right]$.

Conditions were recently announced $\left[\mathbf{P}_{\mathbf{4}}\right]$ for a GO space to be orderable when the closure of its set of pseudogap points is scattered.

One might believe that if a GO space has enough isolated points, an admissible order can be constructed by throwing sequences of order type $\omega_{0}$ or $\omega_{0}^{*}$ into each pseudogap. However, in $[\mathbf{P}-\mathbf{W}]$ it is shown that the subspace of the lexicographic product $[0,1] \times\{0,1,2\}$ consisting of those points with second coordinate 0 or 1 is not orderable even though its spread equals its cardinality.

2. Definitions. A suborderable space with a given admissible suborder will be called a GO space (instead of "subordered", adopting the convention in the preface of [B-L]).

Received by the editors February 11, 1981 and, in revised form, September 10, 1984. 1980 Mathematics Subject Classification. Primary 54F05, 54D35, 06A05.

Key words and phrases. Totally orderable, suborderable, GO space, scattered, ordered compactification, scattered compactification, length, $Q$-gap, pseudogap. 
For most of the definitions pertaining to order relations, see $\left[\mathbf{P}_{\mathbf{2}}\right]$. Whenever a set has an order relation for a subscript, for instance a ray $(-\infty, a)_{\leqslant}$, the set is to be considered an ordered set ordered by $\leqslant$.

A left gap in a GO space $X$ is a nonempty clopen convex subset which is coinitial in $X$ and has no maximum. A left gap is a left $Q$-gap if there is a discrete set cofinal in the gap. A right gap and right $Q$-gap are defined analogously. A pseudogap is a left gap with a supremum in $X$ or a right gap with an infimum in $X$.

The Dedekind compactification of an ordered space $X$ is an ordered compactification of $X$ defined on p. 4 in [J].

Let $\langle X, \leqslant\rangle$ be a scattered GO space, and let $Y \subseteq X$. A linear ordering $\propto$ of $Y$ is $L$-nice ( $R$-nice, respectively) iff (1) it generates the relative topology on $Y$ as a subspace of $X$; (2) the Dedekind compactification of $\langle Y, \underline{\propto}\rangle$ is scattered; and (3) $\underline{\propto}-\min (Y)(\underline{\propto}-\max (Y)$, resp.) exists and is equal to $\leqslant-\min (Y)(\leqslant-\max (Y)$, resp.) if the latter exists. $Y$ is nicely orderable iff it admits both an $L$-nice and an $R$-nice ordering. (Note that the definition in $\left[\mathbf{P}_{\mathbf{3}}\right]$ of a single nice order with both a first and a last point had to be modified since, for example, there is no admissible order on the space $\omega_{1}$ allowing two endpoints.) Both $L$-nice and $R$-nice orderings are required on a space since, in Lemma 2 , each member in a disjoint collection of convex sets is reordered, leaving a specified endpoint of the member fixed.

3. Result. The proof of the result involves a complex series of reorderings. So, most of the proof is broken down into Lemmas 1-3, some of which are technical. Basically, in Lemma 2 and the Theorem, $X$ is partitioned into convex subsets. A nice order is found for each of these convex sets. Then the order relation among the convex sets is rearranged to induce a nice order on the entire space.

LEMMA 1. If $X$ is suborderable and has a discrete cover by nonempty clopen nicely orderable subsets, then $X$ is nicely orderable.

To facilitate the construction of an $L$-nice order, the $L$-nice analogue of the lemma is given in a more technical form. An $R$-nice order is found similarly.

LEMMA 1'. Suppose each $\left\langle X_{i}, \leqslant_{i}\right\rangle(i \in I)$ is an ordered scattered space with a first element. Then there is a function $\phi: I \rightarrow 2$, and there is a linear ordering $\propto$ of $I$ such that if $X$ is the disjoint union $\sum\left\{X_{i}: i \in I\right\}$ as a topological space, then $X$ is orderable by the ordering $\unlhd$ defined as follows: given $x, y \in X, x \unlhd y$ iff either

(i) $x \in X_{i}, y \in X_{j}$, and $i \propto j$; or

(ii) $x, y \in X_{i}, x \leqslant{ }_{i} y$, and $\phi(i)=0$; or

(iii) $x, y \in X_{i}, y \leqslant{ }_{i} x$, and $\phi(i)=1$.

Moreover, given $i_{0} \in I$ where $\leqslant_{i_{0}}-\max \left(X_{i_{0}}\right)$ exists, we may choose $\propto$ and $\phi$ so that $i_{0}=\underline{\propto}-\min (I)$ and $\phi\left(i_{0}\right)=0$, so that $\unlhd-\min (X)=\leqslant_{i_{0}}-\min \left(X_{i_{0}}\right)$. Finally, if each ordered space $\left\langle X_{i}, \leqslant_{i}\right\rangle$ has a scattered Dedekind compactification, so does $\langle X, \unlhd\rangle$.

Note. By definition the first point under any condition of a GO space must be the first point under an $L$-nice order. In Lemma $1^{\prime}$ we actually need for any $i_{0} \in I$ that $\unlhd-\min (X)=\leqslant_{i_{0}}-\min \left(X_{i_{0}}\right)$. So if $\leqslant_{i_{0}}-\min \left(X_{i_{0}}\right)$ does not exist, then there are adjacent points $u$ and $v$ in $\left\langle X_{i_{0}}, \leqslant_{i_{0}}\right\rangle$ where $u \leqslant_{i_{0}} v$. Let $X_{i_{0}}^{\prime}=(-\infty, u]_{\leqslant_{0}}, p$ be a "new" point 
not in $I, X_{p}^{\prime}=[v, \infty)_{\leqslant_{0}}, \leqslant_{i_{0}}^{\prime}$ be $\leqslant_{i_{0}}$ restricted to $X_{i_{0}}^{\prime}, \leqslant_{p}^{\prime}$ be $\leqslant_{i_{0}}$ restricted to $X_{p}^{\prime}$, and for $i \in I-\left\{i_{0}\right\}$ let $X_{i}^{\prime}=X_{i}$ and $\leqslant_{i}^{\prime}=\leqslant_{i}$. Then the hypothesis of Lemma $1^{\prime}$ holds for $\left\{\left\langle X_{i}^{\prime}, \leqslant_{i}^{\prime}\right\rangle: i \in I \cup\{p\}\right\}$ and $\leqslant_{i_{0}}^{\prime}-\max \left(X_{i_{0}}^{\prime}\right)$ exists.

Proof of Lemma $1^{\prime}$. Let $I_{1}=\left\{i \in I: \leqslant i-\max \left(X_{i}\right)\right.$ exists $\}$, and let $I_{0}=I-I_{1}$. Let $\kappa_{e}=\left|I_{e}\right|$ for $e<2$.

Case A. $\kappa_{1} \geqslant \omega$. We may assume that $I_{1}=\left\{\langle\alpha, n\rangle \in \kappa_{1} \times Z: \alpha>0\right.$ or $\left.n \geqslant 0\right\}$ with $i_{0}=\langle 0,0\rangle$ (where $Z$ is the set of integers), and we let $\underline{\propto}_{1}$ be the lexicographic ordering of $I_{1}$. For all $i \in I_{1}$ we put $\phi(i)=0$. Setting $X_{1}=\sum\left\{X_{i}: i \in I_{1}\right\}$, and defining $\unlhd_{1}$ on $X_{1}$ (from $\underline{\propto}_{1}$ and $\phi$, as in the statement of the lemma), we easily verify that $X_{1}$ is orderable by $\unlhd_{1}$ and that $\unlhd_{1}-\min \left(X_{1}\right)=\leqslant_{i_{0}}-\min \left(X_{i_{0}}\right)$.

Subcase 1. $\kappa_{0}<\omega$. Identify $I_{0}$ with $\kappa_{0}$, taking $\underline{\propto}_{0}$ to be the usual ordering of $\kappa_{0}$. Define $\phi$ on $I_{0}$ by $\phi(\alpha)=0$ iff $\alpha$ is odd.

Subcase 2. $\kappa_{0} \geqslant \omega$. Identify $I_{0}$ with $\kappa_{0} \times Z$, taking $\underline{\propto}_{0}$ to be the lexicographic order on $I_{0}$. Define $\phi$ restricted to $I_{0}$ by $\phi(\langle\alpha, n\rangle)=0$ iff $n$ is even.

Case B. $\kappa_{1}<\omega$. Assume that $I_{1}=\kappa_{1}$ and that $i_{0}=0 . \underline{\varrho}_{1}$ is now the usual ordering of $\kappa_{1}$, and again we put $\phi\left[I_{1}\right]=\{0\}$, defining $X_{1}$ and $\unlhd_{1}$ as in Case A. The same conclusions can be drawn.

Subcase 1. $\kappa_{0}<\omega$. Assume $I_{0}=\kappa_{0}$, taking $\underline{\varrho}_{0}$ to be the usual ordering of $\kappa_{0}$. Define $\phi$ on $I_{0}$ by $\phi(\alpha)=0$ iff $\alpha$ is even.

Subcase 2. $\kappa_{0} \geqslant \omega$. Identify $I_{0}$ with $\left\{\langle\alpha, n\rangle \in \kappa_{0} \times Z: \alpha>0\right.$ or $\left.n \geqslant 0\right\}$, again taking $\underline{\propto}_{0}$ to be the lexicographic order on $I_{0}$. Define $\phi$ on $I_{0}$ by $\phi(\langle\alpha, n\rangle)=0$ iff $n$ is even.

Now, for all cases let $\unlhd_{0}$ be defined on $X_{0}$ from $\underline{\propto}_{0}$ and $\phi$ as usual, where, of course, $X_{0}=\Sigma\left\{X_{i}: i \in I_{0}\right\} ; \unlhd_{0}$ has no pseudogaps-only gaps and jumps-so it generates the topology of $X_{0}$.

Finally, let $\underline{\propto}=\underline{\propto}_{1} \cup \underline{\propto}_{0} \cup\left(I_{1} \times I_{0}\right)$ and define $\unlhd$ from $\underline{\propto}$ and $\phi$. (Thus, $\left\langle X_{1}, \unlhd_{1}\right\rangle$ and $\left\langle X_{0}, \unlhd_{0}\right\rangle$ are initial and final segments, respectively, of $\langle X, \unlhd\rangle$.) Clearly, $X$ is orderable by $\unlhd$ unless there is a pseudogap "between" $X_{1}$ and $X_{0}$; but the choice of $\phi$ ensures that $\left\langle X_{1}, X_{0}\right\rangle$ defines either a jump (if $\kappa_{1}<\omega$ ) or a gap (if $\left.\kappa_{1} \geqslant \omega\right)$.

In each case it is rather easy to see that if each $\left\langle X_{i}, \leqslant_{i}\right\rangle(i \in I)$ has a scattered Dedekind compactification, then so has $\langle X, \unlhd\rangle$, since any "new" gaps are ordered essentially as a suborder of $\langle I, \underline{\propto}\rangle$, which is scattered.

LEMMA 2. Let $\langle X, \leqslant\rangle$ be a scattered GO space of length $\lambda+1$, and suppose that every scattered GO space of length $\leqslant \lambda$ is nicely orderable. Then $X$ is nicely orderable.

Proof. By Lemma 1 we may assume $\left|X^{(\lambda)}\right|=1$, since Ind $X=0$ and $X$ is collectionwise Hausdorff implies $X$ is the disjoint union of clopen subsets each of whose $\lambda$-derived set is a singleton. Let $\{x\}=X^{(\lambda)}$.

If $\lambda=0$, then $X$, being a singleton, is obviously nicely orderable, so assume that $\lambda>0$. By the induction hypothesis and Lemma 1 , we need only show some clopen neighborhood of $x$ is nicely orderable. We first define on $(-\infty, x]$, or on some final clopen segment of it, an $L$-nice order $\unlhd_{1}$ with $x$ its greatest element.

Case A. $x \in \operatorname{cl}(-\infty, x)$. Let $\left\langle y_{\alpha}: \alpha<\kappa\right\rangle$ be a strictly increasing sequence of 
isolated points converging up to $x$, where $\kappa$ is regular. Let $I_{0}=\left(-\infty, y_{0}\right)$ and for $0<\alpha<\kappa, I_{\alpha}=\bigcap\left\{\left[y_{\beta}, y_{\alpha}\right): \beta<\alpha\right\}$ (so that $I_{\alpha+1}=\left[y_{\alpha}, y_{\alpha+1}\right.$ ) for each $\alpha<\kappa$ ). Each $I_{\alpha}$ is a scattered GO space of length $\leqslant \lambda$, so by hypothesis it admits an $L$-nice order $\unlhd_{\alpha}$. These orders induce with $\leqslant$ an ordering $\unlhd_{1}^{\prime}$ of $(-\infty, x]_{\leqslant}: x$ is the greatest element, and if $u \in I_{\alpha}$ and $v \in I_{\beta}$, then $u \unlhd_{1}^{\prime} v$ iff $\alpha<\beta$, or $\alpha=\beta$ and $u \unlhd_{\alpha} v$. Moreover, $\unlhd_{1}^{\prime}$ generates the topology on $(-\infty, x]_{\leqslant}$, except possibly for left pseudogaps of the form $\left(-\infty, y_{\alpha}\right)_{\unlhd_{1}^{\prime}}$ (with $\left.0<\alpha<\kappa\right)$ or $\left(-\infty, y_{\alpha}^{-}\right)_{\unlhd_{1}^{\prime}}$ where $\alpha<\kappa$ is a limit ordinal and $y_{\alpha}^{-}=\leqslant-\min \left(I_{\alpha}\right)$. Let $B$ be the set of such "bad" points $y_{\alpha}$ or $y_{\alpha}^{-}$.

Subcase 1. $B$ is not cofinal in $(-\infty, x)$. Choose $\alpha<\kappa$ so that $y<y_{\alpha}$ for each $y \in B$. Clearly the clopen interval $\left[y_{\alpha}, x\right]_{\leqslant}$admits $\unlhd_{1}^{\prime}$ as an $L$-nice ordering. (Note that $\left.\left[y_{\alpha}, x\right]_{\leqslant}=\left[y_{\alpha}, x\right]_{\unlhd_{1}^{\prime}}\right)$ Let $\unlhd_{1}=\unlhd_{1}^{\prime}$.

Subcase 2. $B$ is cofinal in $(-\infty, x)$. By choice of $\kappa$ we may enumerate $B$ as $\left\{y_{\alpha}^{\prime}\right.$ : $\alpha<\kappa\}$ in strictly increasing order. Let $J_{0}=\left(-\infty, y_{0}^{\prime}\right)$ and, for each $\alpha<\kappa, J_{\alpha}=$ $\bigcap\left\{\left[y_{\beta}^{\prime}, y_{\alpha}^{\prime}\right): \beta<\alpha\right\}$. Note (for $\alpha$ nonlimit) $J_{\alpha}$ is a (clopen) union of sets of the form $I_{\gamma}$. Define a function $\phi: \kappa \rightarrow 2$ as follows. Given $\alpha \in \kappa$, write $\alpha=\eta+n$, where $\eta$ is 0 or a limit ordinal, and $n \in \omega$; then $\phi(\alpha)=0$ iff $n$ is even. Now define a linear ordering $\unlhd_{1}$ on $(-\infty, x]$ with $x$ as its greatest element using $\unlhd$ and $\phi$ : if $u \in J_{\alpha}$ and $v \in J_{\beta}$, then $u \unlhd_{1} v$ iff either $\alpha<\beta$; or $\alpha=\beta$ and either $\phi(\alpha)=0$ and $u \unlhd v$, or $\phi(\alpha)=1$ and $v \unlhd u$. It is easy to check that $\unlhd_{1}$ is an $L$-nice ordering of $(-\infty, x)_{\leqslant}$. (Compare with Lemma 1'.)

Case B. $x \notin \operatorname{cl}(-\infty, x)$. Then $\{x\}$ is a final clopen segment of $(-\infty, x]$. Let $\unlhd_{1}=\{\langle x, x\rangle\}$.

By an analogous argument there is an $L$-nice order $\unlhd_{2}$ on $[x, \infty)_{\leqslant}$or on some initial clopen segment of it.

Then $\unlhd_{1} \cup \unlhd_{2}$ is an $L$-nice order on a clopen neighborhood of $x$. So $\langle X, \leqslant\rangle$ admits an $L$-nice ordering.

The argument that $\langle X, \leqslant\rangle$ admits an $R$-nice ordering is, of course, entirely analogous.

Lemma 3. Let $\langle X, \leqslant\rangle$ be a scattered GO space of length $\lambda$, where $\lambda$ is a limit ordinal. Let $b X$ be the greatest ordered compactification of $X$, i.e., the one whose growth is order-isomorphic to the set of gaps of $X$. Let $S$ be the set of all non-Q-gaps $A$ of $X$ (viewed as a subset of $b X$ ) such that $A$ is a limit point of $A^{(\xi)}$ for each $\xi<\lambda$. Then $S$ is a discrete subset of $b X$, and $X \cap \operatorname{cl}_{b X} S=\varnothing$. (Note the dual role above of $A: A$ as $a$ limit point is considered a point of $b X-X$, while for $A^{(\xi)}$ it is considered a subset of $X$.)

Proof. Clearly

$$
\begin{aligned}
X \cap \mathrm{cl}_{b X} S \subseteq X \cap \bigcap_{\xi<\lambda} \mathrm{cl}_{b X} X^{(\xi)}=\bigcap_{\xi<\lambda}\left(X \cap \mathrm{cl}_{b X} X^{(\xi)}\right) \\
=\bigcap_{\xi<\lambda} \operatorname{cl}_{X} X^{(\xi)}=\bigcap_{\xi<\lambda} X^{(\xi)}=X^{(\lambda)}=\varnothing
\end{aligned}
$$

Now suppose there exists a cluster point $u \in S$. Then there would be a monotone sequence $\left\{u_{\alpha}\right\}_{\alpha<\eta}$ in $S$ converging to $u$. For each $\alpha<\eta$ we can choose $x_{\alpha} \in X$ 
between $u_{\alpha}$ and $u_{\alpha+1}$. Then $\left\{x_{\alpha}\right\}_{\alpha<\eta}$ converges to $u$, and since $u$ is a non-Q-gap of $X$, there is a nonzero limit ordinal $\xi<\eta$ such that $\left\{x_{\alpha}\right\}_{\alpha<\xi}$ converges to a point $x \in X$. But then $\left\{u_{\alpha}\right\}_{\alpha<\xi}$ converges to $x$. So $x \in \mathrm{cl}_{b X} S$, which cannot happen.

THEOREM. Let $X$ be a scattered suborderable space. Then $X$ is nicely orderable.

Proof. Let $\leqslant$ be an admissible suborder on $X$. By Lemma 2 we need only consider the case in which the length of $X$ is a limit ordinal $\lambda$, and each scattered GO space of length $<\lambda$ is nicely orderable. Let $b X$ and $S$ be as in Lemma 3.

If $S=\varnothing$, then the proof of the nice orderability of $X$ follows the proof below of the nice orderability of $I_{\alpha}$. So let $S \neq \varnothing$. By Lemmas 1 and 3 we may assume $S=\{u\}$ and $u$ is an end gap of $X$, since Ind $b X=0$ and $b X$ is collectionwise Hausdorff implies $b X$ is the disjoint union of clopen subsets each of which contains one point of $S$.

There is a monotone, say increasing, sequence $\left\{y_{\alpha}\right\}_{\alpha<\kappa}$ of isolated points in $X$ converging to the end gap $u$. Let $I_{\alpha}=\bigcap\left\{\left[y_{\beta}, y_{\alpha}\right): \beta<\alpha\right\}$ for each $\alpha<\kappa$. Proceeding as in Case $\mathrm{A}$ of the proof of Lemma 2, the nice orderability of $X$ will follow from that of the $I_{\alpha}$ 's. So fix $\alpha<\kappa$. If $A \subseteq I_{\alpha}$ is a non- $Q$-gap of $I_{\alpha}$, then $A \in b X-X$, and there is a $\xi<\lambda$ such that $b X-\operatorname{cl}_{b X} A^{(\xi)}$ is an open neighborhood of $A$ in $b X$. That is, $X-\mathrm{cl}_{b X} A^{(\xi)}$ is an open set of $X$ covering the gap $A$. (Note that $A \notin A-A^{(\xi)}$, and, in general, $A^{(\xi)}$ is not closed in $b X$.) Then $\left\{I_{\alpha}-I_{\alpha}^{(\beta)} \mid \beta<\lambda\right\}$ is an open cover of $I_{\alpha}$ which covers the non- $Q$-gaps of $I_{\alpha}$ as well. The argument of Gillman and Henriksen [G-H] that an ordered space is paracompact iff all its gaps are $Q$-gaps shows that the above cover has a locally finite open refinement, $F$, which-since Ind $X=0$-can actually be chosen to be a clopen partition of $I_{\alpha}$. (The fact that $I_{\alpha}^{*}=I_{\alpha} \cup$ (all non- $Q$-gaps of $I_{\alpha}$ ) may not be paracompact does not affect the argument here.) Each memeber of $F$ evidently has length $<\lambda$ and is therefore, by hypothesis, nicely orderable. Then by Lemma $1, I_{\alpha}$, and hence $X$, is nicely orderable.

\section{REFERENCES}

[B-L] H. R. Bennett and D. J. Lutzer (Editors), Topology and order structures. Part I, Tract 142, Math Centre Tracts, Amsterdam, 1981.

[G-H] L. Gillman and M. Henricksen, Concerning rings of continuous functions, Trans. Amer. Math. Soc. 77 (1954), 340-362.

[J] I. Juhasz, Cardinal functions in topologv, Math. Centre Tracts, Amsterdam, 1971.

$\left[\mathbf{P}_{1}\right]$ S. Purisch, Strongly scattered spaces and orderability, Notices Amer. Math. Soc. 23 (1976), A-183.

$\left[\mathbf{P}_{\mathbf{2}}\right]$, The orderability and suborderability of metrizable spaces, Trans. Amer. Math. Soc. 226 (1977), 59-76.

$\left[\mathbf{P}_{3}\right]$, Scattered compactifications and the orderability of scattered spaces, Topology Appl. 12 (1981), 83-88.

$\left[\mathbf{P}_{4}\right]$, The orderability of $\mathrm{GO}$ spaces whose pseudogaps are far or few between, Abstracts Amer. Math. Soc. 4 (1983), \#83T-54-248.

[P-W] S. Purisch and E. Wattel, Non orderability of suborderable spaces with many pseudogaps, Topology and Order Structures. Part I, Tract 142, Math. Centre Tracts, Amsterdam, 1981, pp. 17-25.

[T] R. Telgarsky, Total paracompactness and paracompact dispersed spaces, Bull. Acad. Polon. Sci. Sér. Sci. Math. Astronom. Phys. 16 (1968), 567-572.

Mathematical Institute, ČSAV, Žıtná 25, 15567 Praha 1, Czechoslovakia

Current address: Mathematical Institute of the Hungarian Academy of Sciences, Realtanoda, U. 13-15, Budapest, Hungary H-1053 